\title{
Forecasting Charging Demand of Electric Vehicles Using Time-Series Models
}

\author{
Yunsun Kim (D) and Sahm Kim *(D) \\ Department of Applied Statistics, Chung-Ang University, Seoul 06974, Korea; kys2125@cau.ac.kr \\ * Correspondence: sahm@cau.ac.kr; Tel.: +82-2-820-5225
}

\section{check for}

updates

Citation: Kim, Y.; Kim, S. Forecasting Charging Demand of Electric Vehicles Using Time-Series Models. Energies 2021, 14, 1487. https://doi.org/ $10.3390 /$ en14051487

Academic Editor: Kari Tammi

Received: 9 February 2021

Accepted: 7 March 2021

Published: 9 March 2021

Publisher's Note: MDPI stays neutral with regard to jurisdictional claims in published maps and institutional affiliations.

Copyright: (C) 2021 by the authors. Licensee MDPI, Basel, Switzerland. This article is an open access article distributed under the terms and conditions of the Creative Commons Attribution (CC BY) license (https:// creativecommons.org/licenses/by/ $4.0 /)$.

\begin{abstract}
This study compared the methods used to forecast increases in power consumption caused by the rising popularity of electric vehicles (EVs). An excellent model for each region was proposed using multiple scaled geographical datasets over two years. EV charging volumes are influenced by various factors, including the condition of a vehicle, the battery's state-of-charge (SOC), and the distance to the destination. However, power suppliers cannot easily access this information due to privacy issues. Despite a lack of individual information, this study compared various modeling techniques, including trigonometric exponential smoothing state space (i.e., Trigonometric, BoxCox, Auto-Regressive-Moving-Average (ARMA), Trend, and Seasonality (TBATS)), autoregressive integrated moving average (ARIMA), artificial neural networks (ANN), and long short-term memory (LSTM) modeling, based on past values and exogenous variables. The effect of exogenous variables was evaluated in macro- and micro-scale geographical areas, and the importance of historic data was verified. The basic statistics regarding the number of charging stations and the volume of charging in each region are expected to aid the formulation of a method that can be used by power suppliers.
\end{abstract}

Keywords: electric vehicle; charging demand; charging stations; TBATS; ARIMA; ANN; LSTM

\section{Introduction \\ 1.1. Background}

The advent of electric vehicles (EVs) in the 19th century [1,2] has since posed a growing challenge to the current automobile industry. Battery limitations and high cost initially discouraged the use of commercial EVs. However, increasing environmental protection and global-warming concerns have led to a significant rise in the demand for EVs. Consequently, considerable research and development have facilitated significant progress, thereby overcoming several issues associated with EV batteries. These advances have allowed the EV market to compete with, and in some cases overtake, the combustion-engine automotive industry [3]. Several governments have implemented regulations, incentives, and industry promotions [4] to encourage the effective use of EVs. In addition to economic policies regarding electric vehicles, the supporting infrastructure, including sufficient charging stations and stable power supply in buildings and roads, should be provided.

This study proposes an optimal forecasting model for electric vehicle power suppliers regarding each EV charging unit in a country, city, or single charging station based on realworld data. A previous study [5] reported the superior performance of an autoregressive integrated moving average (ARIMA)-based decoupled forecaster method. Specifically, the power consumption of separate EVs was forecasted instead of the total power consumption. Another study [6] proposed a back-propagation (BP) neural network model that included weather information as an input to improve accuracy. These studies demonstrated that EV charging prediction studies should be conducted individually.

\subsection{Previous Work}

Related research can be classified based on a broad geographical scale. Many studies have forecasted the electricity consumption of a station [7-15], such as a building unit. 
Furthermore, other studies [16,17] have considered cities, where several stations are present. Similarly, a road [18] or several EVs [19-22] can be considered for the prediction of smallscale power consumption. However, simulations in most studies [19,20,23-29] are based on road traffic and not real-world EV electricity consumption data. These studies assume that fossil vehicles will be replaced by EVs in the future and consider current road traffic as a reflection of future EV traffic.

Studies can also be classified based on forecast technique, as either classic statistical techniques or machine learning methods. Classic statistical techniques rely on assumptions; however, they have the advantageous capability of interpreting a factor from estimated values. EV studies commonly use regression modeling [15,19,22,29], ARIMA modeling [5,17,18,30,31], and the Bayesian inference method [22]. Machine learning methods typically offer superior prediction accuracy, where the application of random forests $[13,14,17,27]$ and support vector machines (SVM) [10,11,13,27,31] has been reported. Furthermore, several deep learning technologies have been explored, including artificial neural networks (ANN) [9], convolutional neural networks (CNN) [7,31], recurrent neural networks (RNN) [8,9,29,31], long short-term memory (LSTM) [8,9,29], gated recurrent units (GRU) [8,9], and stacked autoencoders (SAE) [9,31]. Finally, the use of BP neural networks $[6,18,31,32]$ and the pattern sequence-based forecasting (PSF) algorithm [30] has been reported.

Due to the lack of real-world data on EV charging volumes, some simulation studies substitute current road traffic data. These algorithms are typically based on assumption that charging events and amounts are determined by the state-of-charge (SOC) at the arrival time at a destination. Furthermore, day types such as holidays and weekends should be considered when determining road traffic $[9-11,14,17,23,27,28]$. Air conditioning and heating appliances greatly influence the total energy usage of a car, and battery drainage occurs more quickly at lower temperatures. Thus, seasonal and weather information has also been used in several studies [6-11,23,28,29]. In addition, factors such as the SOC, type of car, and battery charging time have been previously considered [10,14,21,23,26-29]. The daily driving patterns and distance information of an individual driver may be obtained using questionnaire surveys or sampling $[5,19,21,25,26,31]$. However, EV scheduling relies on overcoming privacy and security issues before accessing driver and vehicle information [33]. The main concern in this regard is the exploitation of private information for public and commercial purposes, and this is a difficult issue for a power provider to overcome.

Taken together with previous work, our previous work [34] demonstrates the effectiveness of forecasting peak load demand for a building using statistical and artificial intelligence (AI)-based models under various scenarios including exogenous variables. From the results, we find that the ANN model gave the lowest error and showed robustness compared to the statistical time series models. However, the ARIMA model was also valuable in interpreting the coefficients with exogenous variables. The Trigonometric, Box-Cox, Auto-RegressiveMoving-Average (ARMA), Trend, and Seasonality (TBATS) model was superior to other exponential smoothing methods, and it is advisable to apply it when information about the external variables is not offered. Therefore, the TBATS model, ARIMA model, and ANN models were selected to compare their performances. Additionally, we decided to consider the LSTM model, which includes a memory cell for long periods.

\subsection{Contributions}

Due to data privacy issues, this study proposes a daily EV electricity charge forecast technique based on past data, special day indicators, and weather. To ensure the applicability of this forecasting approach based on real-world data and not simulation data, three geographical scales were considered, namely a single station, a city, and a country. It provides energy policy legislators and energy suppliers with planning and EV manufacturers with a new business model. Additionally, with the geographical results, the purpose of our study is to be restrictive evidence in relaxing regulatory restrictions in the privacy issue. Various time-series techniques were compared, including the trigonometric exponential smoothing state space (i.e., TBATS) and ARIMA models, and machine learning techniques such as 
ANN and LSTM modeling. The robustness of the approach was ensured by evaluating the accuracy of forecasts ranging from one day to one month in advance. This provided an indication of which models were appropriate for short-term and mid-term predictions.

ARIMA, ANN, and LSTM are multivariate models that can incorporate day indicators and weather. The performances of these models were compared with the univariate TBATS models, which were based solely on past values, thereby demonstrating whether exogenous variables contribute to prediction accuracy. Some researchers believe that a longer data history is more helpful in the modeling and prediction of future values. This belief was verified in all the models at different geographical scales by comparing the use of reference data histories at 3,6 , and 18 months.

\section{Forecasting Methods}

2.1. Trigonometric, Box-Cox, Auto-Regressive-Moving-Average (ARMA), Trend, and Seasonality (TBATS) Model

De Livera et al. [35] proposed modified state-space models for exponential smoothing to overcome the issues related to broader seasonal pattern variation and to handle correlated errors. Furthermore, the model was restricted to linear homoscedasticity to address the nonlinearity issue. However, the Box-Cox transformation was used for some types of nonlinearity and is defined as follows:

$$
\begin{gathered}
y_{t}^{(\omega)}=\left\{\begin{array}{c}
\frac{y_{t}^{\omega}-1}{\omega}, \omega \neq 0 \\
\log \left(y_{t}\right), \omega=0
\end{array}\right. \\
y_{t}^{(\omega)}=l_{t-1}+\phi b_{t-1}+\sum_{i=1}^{T} S_{t-m_{1}}^{(i)}+d_{t} \\
l_{t}=l_{t-1}+\phi b_{t-1}+\alpha d_{t} \\
b_{t}=(1-\phi) b+\phi b_{t-1}+\beta d_{t} \\
S_{t}^{(i)}=S_{t-m_{i}}^{(i)}+\gamma_{i} d_{t} \\
d_{t}=\sum_{i=1}^{p} \varphi_{i} d_{t-i}+\sum_{i=1}^{q} \theta_{i} \varepsilon_{t-i}+\varepsilon_{t}
\end{gathered}
$$

where $y_{t}^{(\omega)}$ is the Box-Cox transformed observation of the actual demand (kilowatts) for parameter $(\omega)$ at time $t(t=1,2, \ldots, T) ; l_{t}$ is local-level data; $b$ is the long-term trend; and $b_{t}$ is the short-term trend within time $t$, where the value of $b_{t}$ finally converges on $b$ and not zero; $\phi$ is a damping parameter for the trend; $d_{t}$ is a series of ARMA models with orders $(p, q) ; \varepsilon_{t}$ is the random error (white noise) with a mean of zero and constant variance of $\sigma^{2} ; m_{i}$ is the $i$ th seasonal cycle; and $\alpha, \beta$ and $\gamma_{i}$ are the smoothing parameters for $i=1, \ldots, T$.

Non-integer seasonality can be accommodated by incorporating the trigonometric seasonal approach into the model, thereby reducing the calculation time. The final TBATS model involving arguments $\left(\omega, \phi, p, q,\left\{m_{1}, k_{1}\right\},\left\{m_{2}, k_{2}\right\}, \ldots,\left\{m_{T}, k_{T}\right\}\right)$ can be explained as follows:

$$
\begin{gathered}
S_{t}^{(i)}=\sum_{j=1}^{k_{i}} S_{j, t}^{(i)} \\
S_{j, t}^{(i)}=S_{j, t-1}^{(i)} \cos \lambda_{j}^{(i)}+S_{j, t-1}^{*(i)} \sin \lambda_{j}^{(i)}+\gamma_{1}^{(i)} d_{t} \\
S_{j, t}^{*(i)}=-S_{j, t-1} \sin \lambda_{j}^{(i)}+S_{j, t-1}^{*(i)} \cos \lambda_{j}^{(i)}+\gamma_{2}^{(i)} d_{t}
\end{gathered}
$$


where $k_{i}$ is the number of harmonics for the seasonal component; $S_{t}^{(i)}, \gamma_{1}^{(i)}$, and $\gamma_{2}^{(i)}$ are smoothing parameters; $S_{j, t}^{*(i)}$ is the stochastic growth of the $i$ th seasonal component; and $\lambda_{j}^{(i)}=\frac{2 \pi j}{m_{i}} \cdot S_{j, t}^{(i)}$ is the stochastic level of the $i$ th seasonal component by $S_{j, t}^{(i)}$.

\subsection{Autoregressive Integrated Moving Average (ARIMA) Model}

ARIMA [36] is a statistical modeling technique used for time-series analysis. Once the data become stationary, the model comprises nonseasonal orders of $(p, q)$ and seasonal orders of $(P, Q)$. The time-series for series $\left\{y_{t} \mid t=1,2, \ldots, T\right\}$ based on ARIMA $(p, d, q)$ $(P, D, Q)$ can be expressed as follows:

$$
\phi_{p}(l) \Phi_{P}\left(l^{s}\right)(1-l)^{d}\left(1-l^{s}\right)^{D} y_{t}=\theta_{q}(l) \Theta_{Q}\left(l^{s}\right) \varepsilon_{t}
$$

where $y_{t}$ is the actual demand (kilowatts) at time $t(t=1,2, \ldots, T)$ and $\varepsilon_{t}$ is the random error (white noise) during $t$, with a mean of 0 and a constant variance of $\sigma^{2}$. Furthermore, $p$, $d$, and $q$ are integers and orders of the model $\phi_{p}(l)=1-\phi_{1} l-\cdots-\phi_{p} l^{p}$, where $p$ denotes the degree of the nonseasonal autoregressive polynomial $\theta_{q}(l)=1-\theta_{1} l-\cdots-\theta_{q} l^{q}$ and $q$ is the degree of the nonseasonal moving-average polynomial. $P$ denotes the degree of the seasonal autoregressive polynomial for the seasonal operators $\Phi_{P}\left(l^{S}\right)=1-\Phi_{1} l^{S}-$ $\cdots-\Phi_{P} l^{P s}$, and $Q$ denotes the degree of the seasonal moving-average polynomial for $\Theta_{Q}\left(l^{s}\right)=1-\Theta_{1} l^{s}-\cdots-\Theta_{Q} l^{Q s}$. The terms $(1-l)^{d}$ and $\left(1-l^{s}\right)^{D}$ are the nonseasonal and seasonal difference operators for orders $d$ and $D$, respectively, where $s$ is a seasonal cycle.

The potential factors affecting the variability of load demand are considered regressors, e.g., climate or socioeconomic variables. The Reg-ARIMA model is a regression ARIMA model with error terms, where the Reg-ARIMA model with $k$ number of predictors for the series $\left\{y_{t} \mid t=1,2, \ldots, T\right\}$ can be expressed as follows:

$$
\phi_{p}(l) \Phi_{P}\left(l^{s}\right)(1-l)^{d}\left(1-l^{s}\right)^{D}\left(y_{t}-\sum_{i=1}^{k} \beta_{i} \chi_{t i}\right)=\theta_{q}(l) \Theta_{Q}\left(l^{s}\right) \varepsilon_{t}
$$

where $\beta$ is the coefficient of predictor $\chi_{t i}$, which helps interpret the impact of the variable on EV charging demands. In this paper, the temperature and the day indicators were used for the predictors.

\subsection{Artificial Neural Network (ANN)}

Feed-forward ANN models are inspired by the complex connections between the neurons of the human brain. The network of cells carries signals from the body along the axons of neurons, where the signals are transferred between neurons via synapses. Some neurons are structured at birth, while others either grow and mature, or die if considered non-useful [37]. A neural network comprises an input layer of input values, a hidden layer to transform the input values, and an output layer to produce the output values. Weights connect the three layers, while nodes may be included in the middle layer to mix input values during the learning of more complex data. While classic statistical models can provide output directly from an input value as an estimated parameter value, neural network models are referred to as black-box models because certain aspects are difficult to express using an equation, such as the weights in the hidden layer. However, this characteristic of the technique is also advantageous because it allows for the modeling of complex relationships using neurons with nonlinear functions. Furthermore, additional exogenous variables may be included in an ANN-based model.

\subsection{Long Short-Term Memory (LSTM)}

Neural network models (Section 2.3) proceed in the forward direction, assuming that all inputs are independent. RNN modeling involves the application of a current output value while also considering past output information. There are three important connections in RNN: input to hidden layer, hidden to hidden layer, and hidden layer to output layer. 
Here, the weights also go back and forth, connecting the three layers from inputs to hidden, hidden to hidden, and hidden to output. While feedforward networks mainly receive inputs and map them to outputs, the characteristics of cyclic connections of RNNs are designed appropriately for sequential data and many to many sequence modeling can be considered. However, there is a vanishing gradient problem that past information decays quickly in RNN, and LSTM is one of the advanced models used to compensate for this problem. The long short-term memory algorithm was developed in 1997 by Hawkrite and Schmitthuber [38]. Specifically, RNN uses neurons to convey past information while LSTM models effectively retain relatively long sequences using a memory cell structure. LSTM is the most frequently used long-term memory model based on RNN.

\section{Data Analysis}

EV charge data regarding all charging stations in Korea from 2018 to 2019 were obtained from the Korean Ministry of Environment [39]. The original data were organized as individual charging events and included charging time, charging load, and charging station datapoints. The data were aggregated per charging station, city, and country (Table 1) to provide an optimal model for suppliers, regardless of user behavior or car status, while considering privacy issues.

Table 1. Parameter estimations for the classic models at three geographical scales.

\begin{tabular}{cccccccc}
\hline \multirow{2}{*}{ Level } & \multirow{2}{*}{\begin{tabular}{c} 
Number of \\
\cline { 3 - 7 }
\end{tabular}} & \multicolumn{3}{c}{ Number of Charging Events } & \multicolumn{3}{c}{ Energy Usage (kW/Event) } \\
\cline { 3 - 8 } & EV Stations & Min & Max & Mean \pm SD & Min & Max & Mean \pm SD \\
\hline National & 1916 & 1444 & 7824 & $2493 \pm 1494$ & 0.1 & 100 & $16 \pm 9$ \\
City & 155 & 147 & 562 & $298 \pm 74$ & 0.1 & 97 & 32 \\
Station & 1 & 1 & 26 & $15 \pm 8$ & 0.1 & $15 \pm 8$ \\
\hline
\end{tabular}

At a national level, the total of 1916 charging stations in Korea was used for an average of 4293 charging events per day. The city of Seoul has the second-highest proportion of EVs, where 155 charging stations were used for approximately 300 charging events per day. A single charging station in Seoul was evaluated, where its two chargers were used for an average of 10.5 charging events per day. The number of charging events differed according to the geographical area; however, there was no significant difference in the energy per event (14-15 kW). The number of enrolled EVs by 2019 was about 89,918 in the country, and 14,952 in Seoul. Because we were not able to obtain the ID for each car, instead, we could assume that at least 96 drivers share a single station in Seoul city.

A time series plot was prepared for each geographical segment (Figure 1). The country exhibited a clear increase in the total charging capacity due to a growing number of EV vehicles and charging stations. The variation within the series gradually increased over the year. The demand during winter was high and was even higher during the summer. The city exhibited a similar increase over the last two years, where high battery consumption during summer and winter was attributed to the use of heating/cooling appliances and battery shortages. The same trends were observed for the single charging station. 

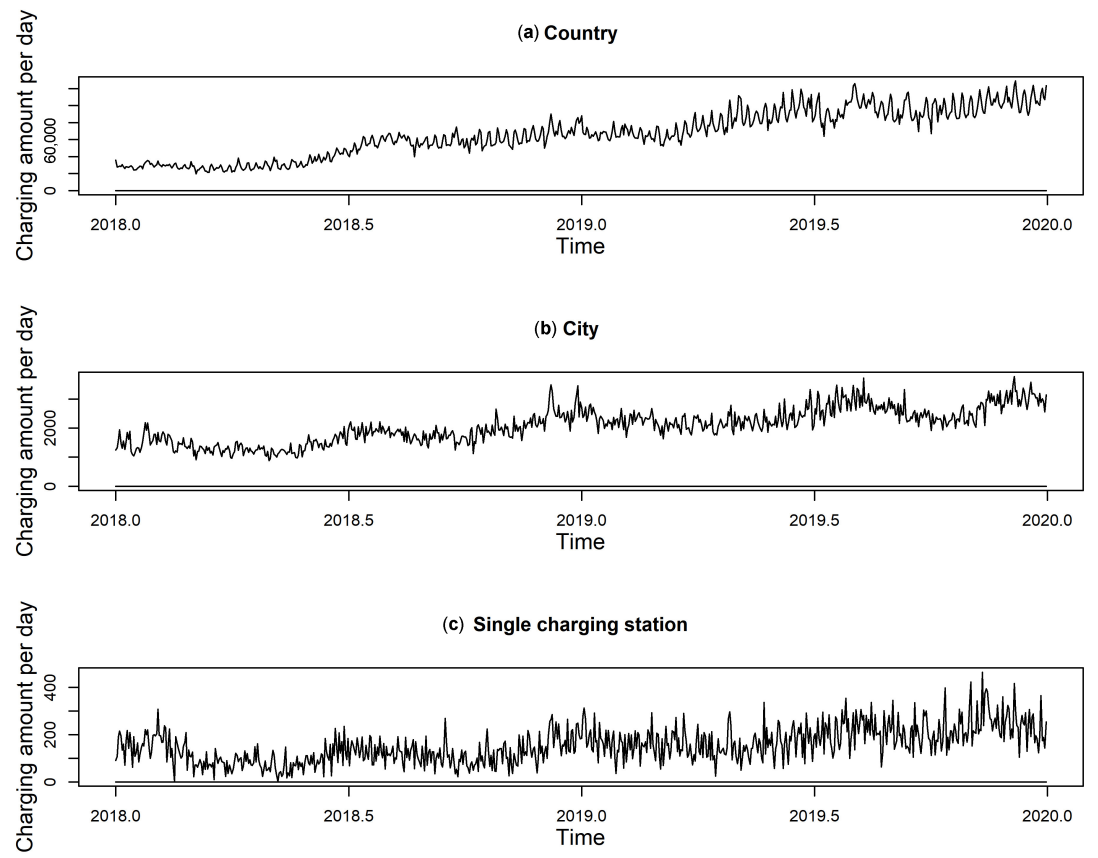

Figure 1. Time series plot of the electric vehicle (EV) charging amount at three geographical scales: (a) country, (b) city, and (c) single charging station. The $y$-axis presents the daily energy used that was calculated by aggregating the multiple charging events (unit: $\mathrm{kW} /$ day). The $x$-axis presents the periods from January 2018 to December 2019.

Table 2 presents the average charged energy per day by weekdays and weekends in four representative months. Since the monthly trends were dramatic, we decided to select four seasons to represent the differences in mean between weekends. The energy used on weekends was much higher in all months and regional scales.

Table 2. Comparisons for charging amount by weekdays and weekends at three geographical scales.

\begin{tabular}{|c|c|c|c|c|c|c|}
\hline \multirow{2}{*}{$\begin{array}{c}\text { Month } \\
\text { Level }\end{array}$} & \multicolumn{3}{|c|}{ January } & \multicolumn{3}{|c|}{ April } \\
\hline & Weekdays & Weekends & Increase Rate (\%) & Weekdays & Weekends & Increase Rate $(\%)$ \\
\hline National & 28,519 & 30,276 & 6.2 & 26,689 & 31,540 & 18.2 \\
\hline City & 1517 & 1540 & 1.5 & 1232 & 1289 & 4.6 \\
\hline Station & 153 & 158 & 3.3 & 77.3 & 97.6 & 26.3 \\
\hline Month & \multicolumn{3}{|c|}{ July } & \multicolumn{3}{|c|}{ October } \\
\hline Level & Weekdays & Weekends & Increase Rate (\%) & Weekdays & Weekends & Increase Rate $(\%)$ \\
\hline National & 52,002 & 59,391 & 14.2 & 56,837 & 62,060 & 9.2 \\
\hline City & 1865 & 2000 & 7.2 & 1770 & 2025 & 14.4 \\
\hline Station & 127 & 143 & 12.6 & 94.3 & 108 & 14.5 \\
\hline
\end{tabular}

Weekly seasonality was evaluated based on a two-month daily time series from November to December 2019 (Figure 2). On the national level, a clear weekly pattern was observed; the most charging during the week occurred on Saturday, followed by Friday and Sunday. Similarly, there was high usage in the city during the weekend; higher usage was observed on weekdays depending on the season. It was more difficult to observe a pattern at the single charging station because of the smaller number of charging events per day; hence, random variability was assumed to be high. Overall, vehicle and driver behaviors were less affected by external variables (e.g., day of the week) as the geographical scale became smaller. 

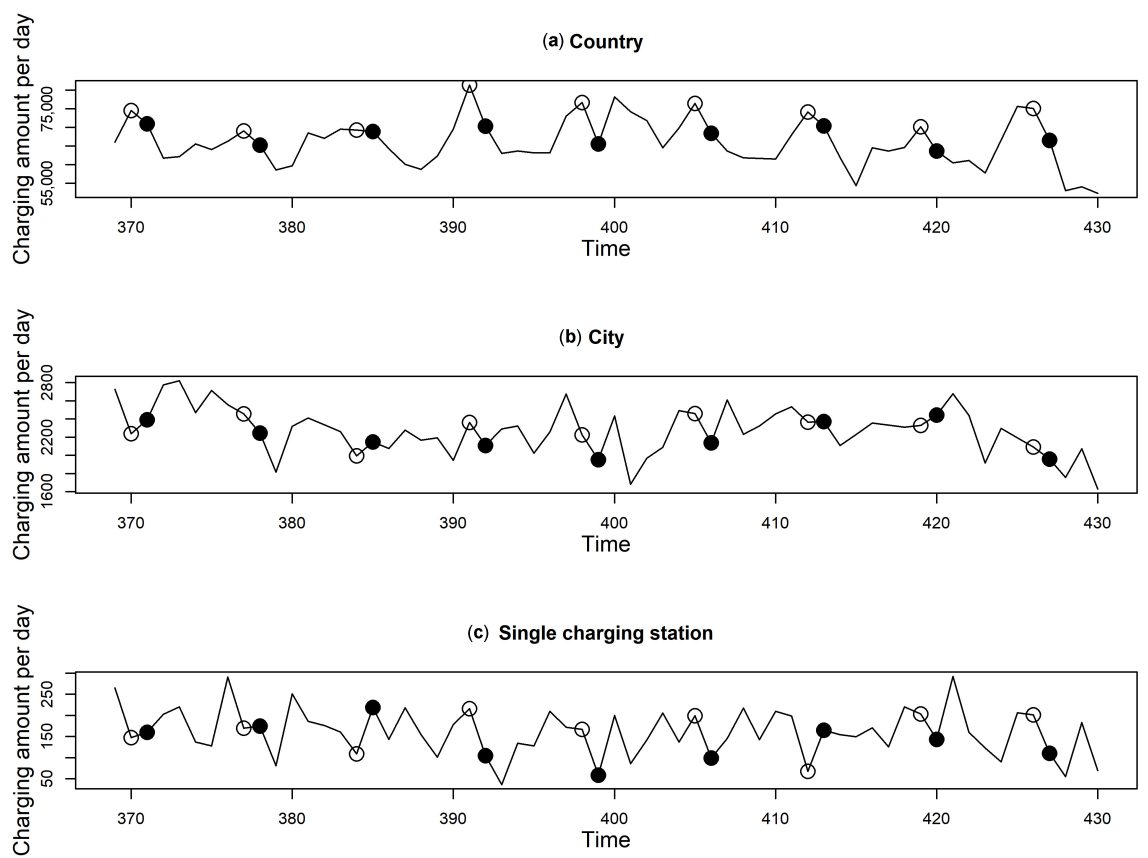

Figure 2. EV charging amount patterns in different seasons at three geographical scales: (a) country, (b) city, and (c) single charging station. White dots represent Saturdays, and black dots represent Sundays. The $y$-axis presents the daily energy used (unit: $\mathrm{kW} /$ day). The $x$-axis presents the periods of two weeks in October.

Temperature, weekends, and holidays were selected as external variables for the ARIMA, ANN, and LSTM models to account for external factors in the series. The temperature $\left({ }^{\circ} \mathrm{C}\right)$ values were converted to heating-degree-day (HDD) and cooling-degree-day (CDD) terms, as expressed in Equations (12) and (13), respectively. Furthermore, the effect of these exogenous variables on the accuracy of regional models was evaluated. Box-Cox transformation (Equation (1)) was performed to ensure the homoscedasticity of the models.

$$
\begin{aligned}
& H D D=\left\{\begin{array}{cc}
18-T_{t}, & \text { if } T_{t} \leq 18 \\
0, & \text { elsewhere }
\end{array}\right. \\
& C D D=\left\{\begin{array}{cc}
T_{t}-24, & \text { if } T_{t} \geq 24 \\
0, & \text { elsewhere }
\end{array}\right.
\end{aligned}
$$

\section{Performance Evaluation}

TBATS, ARIMA, ANN, and LSTM models were tested based on a dataset of 730 data points obtained over two years; the data from 18 months were used for training and those from the remaining 6 months were used for testing. Furthermore, $30 \%$ of the training set was used as a validation set for the machine learning models. The moving-window prediction was applied to provide a range of forecast intervals $(k)$, namely forecasts made one day to one month prior. Each model was fitted for each geographical scale (country, city, and single charging station), and the accuracy was compared. Exogenous variables were used in the ARIMA, ANN, and LSTM models, and the difference in accuracy with and without these variables was compared. Furthermore, the effect of history length on the forecast was investigated by comparing the accuracy using historic data from the past 3,6 , and 18 months.

The forecast package in R software was used; TBATS, ARIMA, and ANN models were performed using the tbats(), arima(), and nnetar() functions, respectively, whereas the LSTM model was calculated using keras. For the ARIMA model and the ANN model, the number of parameters was chosen using a minimum Akaike information criterion, where 
each parameter was estimated in the updated training set. The ANN model included units, number of networks, epochs, and decay set as hyperparameters to determine optimal values. To set the hyperparameters in the LSTM model, search spaces (e.g., units, layers, activation functions, and epochs) were used to identify the hyperparameter values that led to the minimum loss of mean squared error (MSE). Moreover, a regularization method such as weight decay was applied to avoid the overfitting problem in AI models.

The estimated parameters for the time series models based on the 18-month training set are given in Table 3. The influence of the exogenous variables on the ARIMA model was evaluated based on each regressor. The estimated values of the temperature variables, namely CDD and HDD, were found to increase the total power consumption in the all-time series. Furthermore, the weekend variable was found to have a static effect in the country and single charging station forecasts and decreased the accuracy of the city forecast. In addition, the search space and final selection values of the hyperparameters for the machine learning models are given in Table 4.

Table 3. Parameter estimations for the classic models at three geographical scales.

\begin{tabular}{|c|c|c|c|c|c|c|c|c|c|c|}
\hline \multirow[t]{2}{*}{ Model } & \multirow[t]{2}{*}{ Level } & \multicolumn{9}{|c|}{ Estimated Parameters } \\
\hline & & $\omega$ & $\alpha$ & $\varphi_{1}$ & $\varphi_{2}$ & $\varphi_{3}$ & $\varphi_{4}$ & $\varphi_{5}$ & $\theta_{1}$ & $\theta_{2}$ \\
\hline TBATS & $\begin{array}{c}\text { National } \\
\text { City } \\
\text { Station }\end{array}$ & $\begin{array}{l}0.001 \\
0.117 \\
0.607\end{array}$ & $\begin{array}{c}0.434 \\
0.13 \\
0.111\end{array}$ & $\begin{array}{c}0.275 \\
-0.514\end{array}$ & $\begin{array}{c}-0.838 \\
0.215\end{array}$ & 0.005 & -0.347 & -0.359 & $\begin{array}{l}0.028 \\
0.829\end{array}$ & 0.578 \\
\hline & & $\phi_{1}$ & $\theta_{1}$ & $\Phi_{1}$ & $\Theta_{1}$ & & & & & \\
\hline ARIMA & $\begin{array}{c}\text { National } \\
\text { City } \\
\text { Station }\end{array}$ & $\begin{array}{c}0.525 \\
0.515 \\
-0.882\end{array}$ & $\begin{array}{l}-0.878 \\
-0.865\end{array}$ & 0.097 & $\begin{array}{l}-0.999 \\
-0.984 \\
-0.999\end{array}$ & & & & & \\
\hline & & $\phi_{1}$ & $\theta_{1}$ & $\Phi_{1}$ & $\Theta_{1}$ & $\beta_{C D D}$ & $\beta_{H D D}$ & $\beta_{\text {Weekend }}$ & & \\
\hline Reg-ARIMA & $\begin{array}{c}\text { National } \\
\text { City } \\
\text { Station }\end{array}$ & $\begin{array}{c}0.309 \\
0.128 \\
-0.910\end{array}$ & $\begin{array}{l}-0.801 \\
-0.876\end{array}$ & $\begin{array}{l}1.000 \\
0.968\end{array}$ & $\begin{array}{l}-0.975 \\
-0.875\end{array}$ & $\begin{array}{l}0.032 \\
0.024 \\
0.062\end{array}$ & $\begin{array}{l}0.029 \\
0.023 \\
0.272\end{array}$ & $\begin{array}{c}0.389 \\
-0.016 \\
0.471\end{array}$ & & \\
\hline
\end{tabular}

Table 4. Hyperparameters for the machine learning models at three geographical scales.

\begin{tabular}{|c|c|c|c|c|c|c|}
\hline \multirow[t]{2}{*}{ Model } & \multirow[t]{2}{*}{ Level } & \multicolumn{5}{|c|}{ Estimated Hyperparameters } \\
\hline & & Units & Decay & Networks & Epochs & \\
\hline \multirow{4}{*}{ ANN } & National & 50 & 0.85 & 20 & 100 & \\
\hline & City & 50 & 0.90 & 30 & 150 & \\
\hline & Station & 100 & 0.90 & 35 & 50 & \\
\hline & & Units & Decay & Networks & Epochs & \\
\hline \multirow{4}{*}{$\begin{array}{c}\text { ANN } \\
\text { with } \\
\text { regressors }\end{array}$} & National & 100 & 0.90 & 50 & 100 & \\
\hline & City & 150 & 0.85 & 45 & 100 & \\
\hline & Station & 200 & 0.90 & 40 & 50 & \\
\hline & & Units & Decay & Layers & Epochs & Activation Function \\
\hline \multirow{4}{*}{ LSTM } & National & 50 & 0.90 & 1 & 100 & sigmoid \\
\hline & City & 150 & 0.95 & 2 & 200 & tanh \\
\hline & Station & 150 & 0.99 & 3 & 50 & sigmoid \\
\hline & & Units & Decay & Layers & Epochs & Activation Function \\
\hline \multirow{3}{*}{$\begin{array}{c}\text { LSTM } \\
\text { with } \\
\text { regressors }\end{array}$} & National & 150 & 0.90 & 2 & 100 & $\tanh$ \\
\hline & City & 100 & 0.95 & 1 & 100 & sigmoid \\
\hline & Station & 150 & 0.99 & 3 & 100 & tanh \\
\hline
\end{tabular}


The accuracies of the prediction models were compared according to each geographical scale based on mean absolute percentage error (MAPE), which is commonly used to present the error of short-term load forecasting and can be expressed as follows:

$$
M A P E=\frac{100}{n} \sum_{t=1}^{n}\left|\frac{y_{t}-\widehat{y_{t}}}{y_{t}}\right|
$$

where $y_{t}$ is the actual value and $\widehat{y_{t}}$ is the forecast demand at time $t$.

\subsection{Macro-Scale Aggregated Data: National Level}

The one-step-ahead forecasting results using each model based on the national data are given in Table 5. The ARIMA, ANN, and LSTM models that included all the exogenous variables exhibited better performance than the univariate ones. Thus, the robustness and history length were evaluated only in models that included exogenous variables.

Table 5. Accuracy of one-step-ahead forecasting at the national scale.

\begin{tabular}{|c|c|c|c|c|c|c|c|c|c|c|c|c|}
\hline \multirow{2}{*}{ History (Months) } & \multicolumn{3}{|c|}{ TBATS } & \multicolumn{3}{|c|}{ ARIMA } & \multicolumn{3}{|c|}{ ANN } & \multicolumn{3}{|c|}{ LSTM } \\
\hline & 18 & 6 & 3 & 18 & 6 & 3 & 18 & 6 & 3 & 18 & 6 & 3 \\
\hline Univariate & 6.5 & 6.4 & 7.7 & 5.3 & 5.8 & 6.7 & 7.2 & 6.9 & 7.2 & 15.1 & 9.6 & 8.9 \\
\hline Multivariate & & & & 5.0 & 5.4 & 4.7 & 5.7 & 5.4 & 6.9 & 7.0 & 7.1 & 8.7 \\
\hline
\end{tabular}

The rolling forecasting results based on different lengths of historic data are given in Table 6 . The accuracy of the one-day forecast $(k=1)$ using the TBATS, ANN, and LSTM models was higher when a long history was used; however, the ARIMA model exhibited better prediction accuracy when the shortest history ( 3 months) was used. Forecast using all the models for one week ahead $(k=7)$ was inaccurate when only 3 months of historic values were used. The forecast for three weeks ahead $(k=21)$ was generally more accurate as the history length increased, although the best prediction using the LSTM model was achieved when only 3 months history was used. In fact, even the one-month forecast $(k=30)$ using the LSTM model was more accurate when a shorter history length was used. However, the remaining models, namely TBATS, ARIMA, and ANN, performed best when more historic information was included.

Table 6. Accuracy of rolling forecasting with different historical lengths (3, 6, and 18 months) at the national scale.

\begin{tabular}{|c|c|c|c|c|c|c|c|c|c|c|c|c|}
\hline \multirow{2}{*}{ Steps Ahead (K) } & \multicolumn{3}{|c|}{ TBATS } & \multicolumn{3}{|c|}{ ARIMA } & \multicolumn{3}{|c|}{ ANN } & \multicolumn{3}{|c|}{ LSTM } \\
\hline & 18 & 6 & 3 & 18 & 6 & 3 & 18 & 6 & 3 & 18 & 6 & 3 \\
\hline One day $(k=1)$ & 6.5 & 6.4 & 7.7 & 5.0 & 5.4 & 4.7 & 5.7 & 5.4 & 6.9 & 7.0 & 7.1 & 8.7 \\
\hline One week $(k=7)$ & 7.7 & 8.7 & 9.2 & 7.9 & 7.9 & 8.2 & 7.0 & 6.8 & 7.4 & 10.8 & 9.0 & 11.1 \\
\hline Three weeks $(k=21)$ & 9.3 & 9.8 & 11.5 & 9.3 & 10.6 & 13.5 & 7.4 & 9.7 & 8.5 & 11.9 & 12.9 & 6.7 \\
\hline One month $(k=30)$ & 11.0 & 12.4 & 12.9 & 8.3 & 9.3 & 12.6 & 8.2 & 10.9 & 9.4 & 13.0 & 14.0 & 6.3 \\
\hline$K=30 / 1$ ratio & 1.7 & 1.9 & 1.7 & 1.6 & 1.7 & 2.7 & 1.4 & 2.0 & 1.4 & 1.9 & 2.0 & 0.7 \\
\hline
\end{tabular}

The robustness of the models was evaluated by attempting to achieve accurate midterm predictions $(k=30)$ while maintaining good short-term predictions $(k=1)$, where the ratio of the MAPE values at $(k=1)$ and 30 was used. A MAPE ratio greater than 1 indicated that the prediction accuracy was lower in the mid-term than in the short-term. Conversely, a value below 1 indicated that the predictions were more accurate when forecasting in the future. Furthermore, a value closer to 1 indicated higher robustness and consistency.

The one-day forecast $(k=1)$ from the ARIMA model based on 3 months historic information was accurate (MAPE $=4.7 \%)$; however, the accuracy decreased sharply by one month $(k=30)(\mathrm{MAPE}=12.6 \%)$, thereby giving a MAPE ratio of 2.7. The ARIMA model based on 18 months historic information exhibited slightly lower accuracy for the one-day 
prediction $(k=1)(\mathrm{MAPE}=5.0 \%)$ but maintained relatively good accuracy until one month $(k=30)(\mathrm{MAPE}=8.3 \%)$, giving a MAPE ratio of 1.66. Interestingly, the LSTM model based on 3 months historic information exhibited better mid-term prediction accuracy than short-term $($ MAPE ratio $=6.3)$. This was attributed to the memory cells, which maintained long-term memory. Overall, a long history was generally helpful in the TBATS, ARIMA, and ANN models; however, it did not enhance the performance of LSTM.

\subsection{Macro-Aggregated Data: City Level}

The one-step-ahead forecasting results using each model based on the city data are given in Table 7. Both the ARIMA and ANN models performed better when the exogenous variables were included. Thus, the robustness and history length were evaluated only in models that included exogenous variables.

Table 7. Accuracy of one-step-ahead forecasting at the city scale.

\begin{tabular}{|c|c|c|c|c|c|c|c|c|c|c|c|c|}
\hline \multirow{2}{*}{ History (Months) } & \multicolumn{3}{|c|}{ TBATS } & \multicolumn{3}{|c|}{ ARIMA } & \multicolumn{3}{|c|}{ ANN } & \multicolumn{3}{|c|}{ LSTM } \\
\hline & 18 & 6 & 3 & 18 & 6 & 3 & 18 & 6 & 3 & 18 & 6 & 3 \\
\hline Univariate & 8.6 & 8.8 & 8.7 & 8.5 & 9.0 & 9.4 & 9.7 & 10.3 & 9.4 & 14.1 & 10.5 & 10.0 \\
\hline Multivariate & & & & 7.9 & 7.9 & 7.8 & 9.0 & 9.2 & 10.0 & 15.6 & 13.5 & 9.9 \\
\hline
\end{tabular}

The rolling forecasting results based on different lengths of historic data are given in Table 8. The accuracy of the one-day forecast $(k=1)$ of the ANN model was higher with a longer history, whereas a short history was best for the LSTM model. There was no significant difference between the short-term predictions using TBATS and ARIMA; thus, the shorter history provided sufficient information. However, the longer history facilitated better one-week, three-week, and one-month forecasts, even in the TBATS and ARIMA models.

Table 8. Accuracy of rolling forecasting with different historical lengths (3, 6, and 18 months) at the city scale.

\begin{tabular}{|c|c|c|c|c|c|c|c|c|c|c|c|c|}
\hline \multirow{2}{*}{ Steps Ahead (K) } & \multicolumn{3}{|c|}{ TBATS } & \multicolumn{3}{|c|}{ ARIMA } & \multicolumn{3}{|c|}{ ANN } & \multicolumn{3}{|c|}{ LSTM } \\
\hline & 18 & 6 & 3 & 18 & 6 & 3 & 18 & 6 & 3 & 18 & 6 & 3 \\
\hline One day $(k=1)$ & 8.6 & 8.8 & 8.7 & 7.9 & 7.9 & 7.8 & 9.0 & 9.2 & 10.0 & 15.6 & 13.5 & 9.9 \\
\hline One week $(k=7)$ & 9.6 & 9.7 & 10.6 & 8.0 & 8.7 & 8.6 & 10.7 & 9.1 & 10.4 & 17.0 & 18.4 & 17.5 \\
\hline Three weeks $(k=21)$ & 13.0 & 14.7 & 14.3 & 8.4 & 10.7 & 9.3 & 13.8 & 12.5 & 13.1 & 21.4 & 23.1 & 21.6 \\
\hline One month $(k=30)$ & 16.4 & 18.7 & 17.7 & 10.0 & 12.9 & 12.4 & 16.7 & 14.8 & 14.6 & 22.4 & 26.0 & 23.8 \\
\hline$K=30 / 1$ ratio & 1.9 & 2.1 & 2.0 & 1.3 & 1.6 & 1.6 & 1.9 & 1.6 & 1.5 & 1.4 & 1.9 & 2.4 \\
\hline
\end{tabular}

The robustness of the models was evaluated based on the highest performance in short-term predictions, as was determined at the national level. All the ARIMA models exhibited an excellent MAPE $(>8 \%)$ for the one-day forecasts $(k=1)$, regardless of history length. However, the 18-months history was more favorable for long-term predictions. The TBATS model exhibited similar one-day forecast $(k=1)$ performance to that of ARIMA; however, the mid- to long-term predictions were much lower, which led to unsatisfactory robustness. Furthermore, the LSTM model performed unsatisfactorily throughout the city level forecasts, although the short-term prediction was better with a shorter history, as was observed on the national level.

The city unit demonstrated improved predictive power in the time series of aggregated data with exogenous variables. Similar to the national level, the historic data improved the short- to mid-term predictive power in the ARIMA model. Furthermore, the ARIMA model outperformed the LSTM model when a short history was used.

Overall, the ARIMA, TBATS, ANN, and LSTM models exhibited promising results, where the predictive power was more useful in the linear model due to a better fit of the data to the linear function. Information regarding exogenous variables was the best 
fit for ARIMA, better than TBATS, a univariate model. LSTM is a type of RNN with growing popularity. However, it cannot be assumed that LSTM will always outperform feed-forward neural networks. Furthermore, even with the application of decay, it cannot be assumed that a long history will enhance the performance if the role of the LSTM memory cell is excessive.

\subsection{Micro-Data: Single Charging Station}

The one-step-ahead forecasting results using each model based on the data for a single small-scale charging station are given in Table 9. The external variables did not improve the accuracy of the ARIMA model when the history length was 6 to 18 months. However, the exogenous variables worked effectively when 3 months historic data were used. Similarly, the ANN did not exhibit enhanced accuracy when the exogenous variables were considered. However, the results of the LSTM model were satisfactory when exogenous variables were used throughout. Though the models without the exogenous variables were generally more accurate, there was no significant difference. Even in mid-term predictions, only models with the exogenous variables were compared to determine whether the exogenous variables enhanced the forecasting performance on a micro-scale (single charging station).

Table 9. Accuracy of one-step-ahead forecasting at the single charging station scale.

\begin{tabular}{|c|c|c|c|c|c|c|c|c|c|c|c|c|}
\hline \multirow{2}{*}{ History (Months) } & \multicolumn{3}{|c|}{ TBATS } & \multicolumn{3}{|c|}{ ARIMA } & \multicolumn{3}{|c|}{ ANN } & \multicolumn{3}{|c|}{ LSTM } \\
\hline & 18 & 6 & 3 & 18 & 6 & 3 & 18 & 6 & 3 & 18 & 6 & 3 \\
\hline Univariate & 25.6 & 27.6 & 25.9 & 24.6 & 24.8 & 25.8 & 29.5 & 29.5 & 29.8 & 37.3 & 30.5 & 26.9 \\
\hline Multivariate & & & & 25.2 & 24.5 & 20.6 & 31.7 & 32.1 & 29.3 & 23.4 & 21.4 & 24.7 \\
\hline
\end{tabular}

The rolling forecasting results of the TBATS model and the ARIMA, ANN, and LSTM models with exogenous variables are given in Table 10. The accuracy of the one-day forecast $(k=1)$ using the TBATS model was high when more historic information was included; however, the differences between the various history lengths were not significant. Furthermore, the ARIMA and ANN models were most accurate with a short history, whereas the accuracy of the LSTM model was the highest when 6 month historical data were used. The TBATS and ARIMA models were slightly more accurate for one-week, three-week, and one-month forecasts $(k=7,21$, and 30); however, there was no significant difference. Furthermore, the one-week and three-week forecasts $(k=7$, and 21$)$ using the ANN model were accurate when only 3 months historic data were used, whereas the LSTM model was not significantly affected by the history length.

Table 10. Accuracy of rolling forecasting with different historical lengths (3, 6, and 18 months) at the single charging station scale.

\begin{tabular}{ccccccccccccc}
\hline \multirow{2}{*}{ Steps Ahead $(\boldsymbol{K})$} & \multicolumn{3}{c}{ TBATS } & \multicolumn{3}{c}{ ARIMA } & \multicolumn{3}{c}{ ANN } & \multicolumn{3}{c}{ LSTM } \\
\cline { 2 - 12 } & $\mathbf{1 8}$ & $\mathbf{6}$ & $\mathbf{3}$ & $\mathbf{1 8}$ & $\mathbf{6}$ & $\mathbf{3}$ & $\mathbf{1 8}$ & $\mathbf{6}$ & $\mathbf{3}$ & $\mathbf{1 8}$ & $\mathbf{6}$ & $\mathbf{3}$ \\
\hline One day $(\boldsymbol{k}=\mathbf{1})$ & 25.6 & 27.6 & 25.9 & 25.2 & 24.5 & 20.6 & 31.7 & 32.1 & 29.3 & 23.4 & 21.4 & 24.7 \\
One week $(\boldsymbol{k}=\mathbf{7})$ & 25.6 & 27.8 & 26.1 & 24.6 & 27.0 & 25.7 & 30.3 & 31.0 & 29.0 & 24.6 & 20.9 & 25.6 \\
Three weeks $(\boldsymbol{k}=\mathbf{2 1})$ & 25.6 & 30.6 & 26.0 & 23.3 & 32.8 & 26.7 & 28.3 & 44.4 & 26.9 & 26.6 & 28.7 & 30.0 \\
One month $(\boldsymbol{k}=\mathbf{3 0})$ & $\mathbf{2 8 . 9}$ & 37.5 & 29.3 & 25.6 & 27.3 & 25.7 & 32.3 & 52.5 & 35.4 & 24.3 & 24.4 & 23.6 \\
\hline $\boldsymbol{K}=\mathbf{3 0 / 1}$ ratio & 1.1 & 1.4 & 1.1 & 1.0 & 1.1 & 1.2 & 1.0 & 1.6 & 1.2 & 1.0 & 1.1 & 1.0 \\
\hline
\end{tabular}

All the models exhibited MAPE ratios close to 1 . However, their robustness cannot be assumed because the one-step-ahead forecasts of the single EV station were generally less accurate. Thus, a small geographical scale is associated with the difficulty in predicting EV charging based on past values, calendar, and weather effects.

The actual values for two weeks in October 2019 were plotted with the predicted values from each model (Figure 3). The national level exhibited a clear weekly pattern, 
indicating that all the models exhibited good predictive performances. However, the LSTM model tended to underestimate the variance. The weekly pattern was not clear on the city scale, wherein only the ARIMA and ANN models predicted the fluctuation at the peak EV charging times, whereas LSTM showed no significant change in volatility. The plot of the single charging station revealed low overall prediction accuracy, which was attributed to the difficulty in predicting the peak date based on external variables or past values.
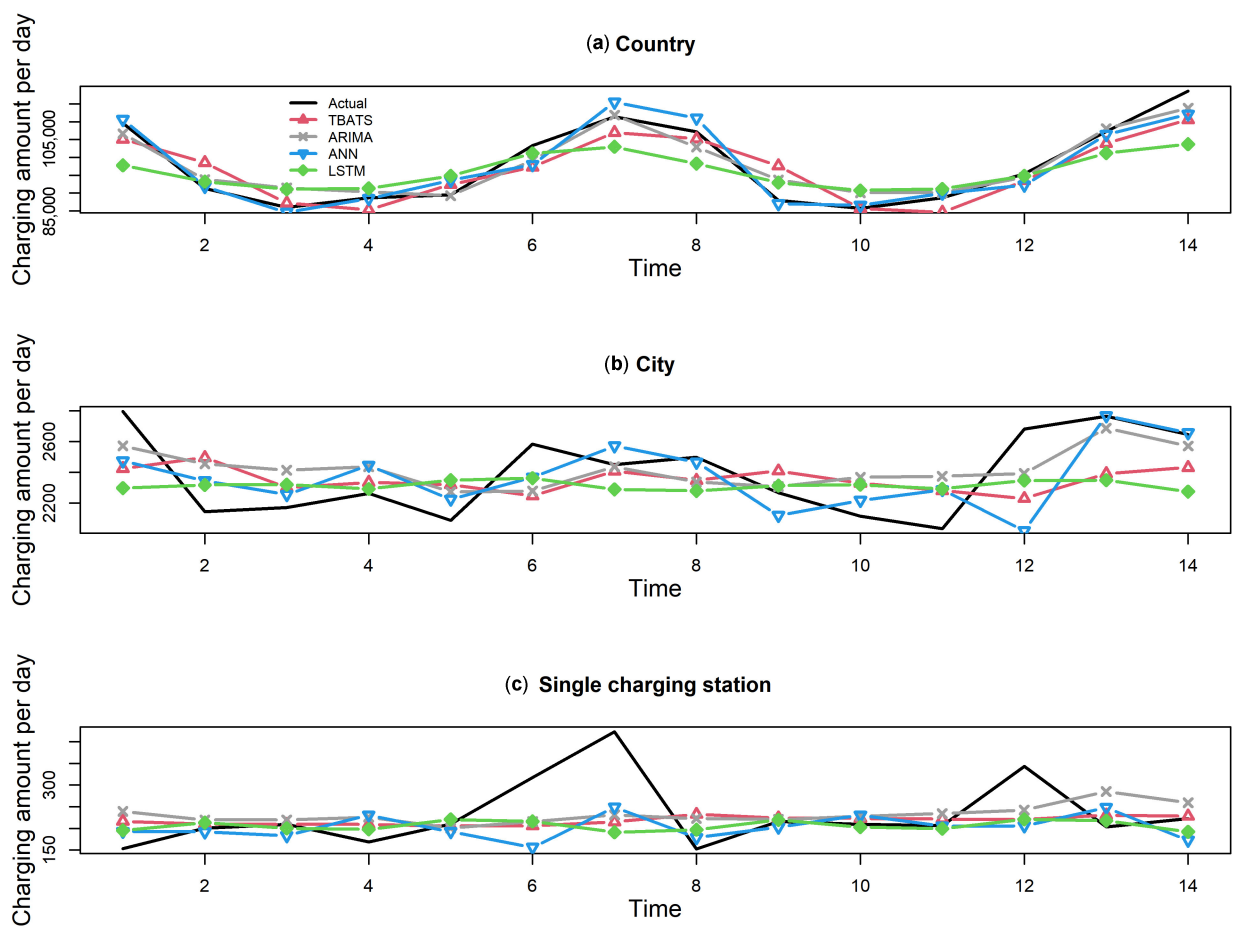

Figure 3. Predicted and actual values for three geographical scales: (a) country, (b) city, and (c) single charging station The $y$-axis presents the daily energy used (unit: $\mathrm{kW} / \mathrm{day}$ ). The $x$-axis presents the day in October 2019.

\subsection{Discussion}

Aggregated data from multiple charging stations on a national and city scale revealed useful patterns for power suppliers, thereby facilitating higher accuracy. The application of exogenous variables in the ARIMA, ANN, and LSTM models (regardless of history length) generally led to higher forecast accuracy on the nation and city levels. However, the small scale of the charging station posed a challenge in forecasting, where only about 10 charging events occurred per day. Thus, exogenous variables did not contribute to the predictions when more than six months of historic data were incorporated into the ARIMA and ANN models. It is worth a try to split the models between the weekday and the weekends due to different energy patterns in macro-scale for the higher performance. Conversely, threemonth historic data paired with the exogenous variables effectively enhanced the predictive power. Despite the clear special day and temperature effect patterns in the aggregated data, the single charging station micro-unit was more affected using individual factors such as driver characteristics, SOC, and car type. Despite these problems, the LSTM model provides a good micro-scale forecast when exogenous variables are used.

A stable EV charging power supply model provides historical data to build a database. A minimum length is typically required when fitting a model; however, this study investigated whether a longer history of past values unconditionally increased prediction accuracy. One-step-ahead forecasting revealed that historical data did not necessarily enhance forecasting power, specifically in the LSTM models, although more historical data did generally facilitate better forecasting power due to improved long-term robustness. 
The negative effect of the historic data on the LSTM model was attributed to those historic data with a small variance that does not provide valuable information for future events with large variance due to the memory cell of the model.

Furthermore, classic and machine learning modeling techniques were compared. It was difficult to apply classic techniques because there are certain assumptions (e.g., stationary state) required to fit a series to the model. Consequently, the machine learning methods were preferred due to easier hyperparameter tuning and superior performance. However, machine learning methods did not always exhibit powerful predictive capabilities. The national and city datasets were linear and patterned macro-units; thus, the classic ARIMA model was the most accurate and robust, followed by TBATS, ANN, and LSTM. Specifically, the ARIMA model is best in the presence of regressor information whereas the TBATS model provided good short-term prediction when exogenous variable information was not given. As mentioned, LSTM did not perform well when a long history was used because the memory cell of the model retained information for too long. However, the LSTM model performed better than the classic and simple micro-data methods in cases with relatively high variability. In general, the differences between the classic and machine learning approaches were not clear in the case of micro-scale data with high variability. The studied EV charging station was adjacent to a highway road and was not in a residential or commercial district. Thus, fewer drivers routinely visited this charging station, thereby leading to weak driver behavioral patterns. Instead, micro-data should be accessed at a customer level, where car type, SOC, traffic volume, and destination scheduling are important factors.

The findings demonstrated that the consideration of exogenous variables generally enhanced the forecast accuracy. Specifically, the aggregated data revealed that calendar and weather information can be used to effectively describe the entire time series, including the original target variables. Assuming that the maintenance of a sufficient database is viable, historical data shows promise in increasing the short- and mid-term predictive power in the ARIMA model. However, three-month historical data is sufficient for accurate mid-term predictions using the LSTM model.

\section{Conclusions}

In response to the growing popularity of EVs, a forecast model for electricity consumption should be established. Previous studies have demonstrated that building separate EV charging forecast models instead of predicting the total conventional power consumption can lead to improved prediction. The factors affecting a single car, such as car type, SOC, drive behavior, and destination, have been considered in previous studies. However, it is difficult for a power supplier to easily incorporate these factors into a predictive model due to privacy issues. Therefore, this study considered predictions based on past values, weather, and day effects as alternatives. The forecasts were divided into national, city, and single charging station patterns, thereby providing insights into the predictability of various regional scales.

This study examined a model that shows the best results when using only past data and public data due to privacy issues. The results were presented in the geographical scales of a nation, city, and station using actual measured data for applicability to other areas. Therefore, analyzing multivariate models of ARIMA, ANN, and LSTM showed higher accuracy than univariate models. However, in single station data, exogenous variables did not significantly influence accuracy because individual behavior is an important factor in determining consumption. Therefore, in order to increase the predictive power in microunits, privacy issues must be resolved.

Next, the robustness was checked for the stable power supply. Is long data always useful at this time? Three scenarios for the history length were compared: $3 \mathrm{~m}, 6 \mathrm{~m}$, and $12 \mathrm{~m}$. As a result, it was found that previous values were unconditionally stable in the short-term forecast, but the past values played an essential role in mid- to long-term forecast. 
Future studies should focus on fitting the same models using past data and future data also for the validation.

Lastly, time-series techniques and machine learning techniques were compared. Machine learning had the advantage of relatively few assumptions for model fitting and easy hyperparameter tuning, but as a result, it did not always show good predictive power. In macro-data with relatively straightforward patterns, the ARIMA model with regressors showed the best results, followed by TBATS, ANN, and LSTM. The TBATS model is expected to be useful when only univariate values are available. The LSTM modelshowed the best performance for micro-data. However, it is still likely that other forecasting methods need to be developed because the influence of individual EV factors is considered significant in a micro-unit.

Several previous studies have reported that past observations play a secondary role. EV distribution and charging are not sufficiently established in certain regions. Therefore, a bottom-up and top-down dominance at the micro-level cannot be established yet. An effective micro-scale EV charging station prediction technique must be further investigated using appropriate simulated or actual data. Moreover, the privacy issues regarding driver information should be resolved to effectively forecast power supply for charging stations within the smart grid market. Exploring the EV charging patterns is important not only for policy legislators and suppliers but also for EV manufacturers because these charging infrastructures certainly attract new-EV buyers as an administrative strategy. At the same time, to solve unstable energy supply planning in micro-scale sites such as charging stations, again, privacy issues need to be discussed and relaxed soon.

Author Contributions: conceptualization, Y.K. and S.K.; methodology, Y.K.; software, Y.K.; validation, Y.K. and S.K.; investigation, Y.K.; writing-original draft preparation, Y.K.; writing-review and editing, S.K.; visualization, Y.K.; supervision, S.K.; project administration, S.K.; funding acquisition, S.K. All authors have read and agreed to the published version of the manuscript.

Funding: This research was funded by Korea Institute of Energy Technology Evaluation and 469 Planning (KETEP) grant number 20199710100060 and the National Research Foundation of Korea (NRF) grant number 2016R1D1A1B01014954.

Conflicts of Interest: The authors declare no conflict of interest.

\section{Abbreviations}

The following abbreviations are used in this manuscript:

$\begin{array}{ll}\text { ANN } & \text { artificial neural networks } \\ \text { ARIMA } & \text { autoregressive integrated moving average } \\ \text { BP } & \text { back-propagation } \\ \text { CDD } & \begin{array}{l}\text { cooling-degree-day } \\ \text { CNN }\end{array} \\ \text { convolutional neural networks } \\ \text { EV } & \text { electric vehicle } \\ \text { GRU } & \text { gated recurrent units } \\ \text { HDD } & \text { heating-degree-day } \\ \text { LSTM } & \text { long short-term memory } \\ \text { MAPE } & \text { mean absolute percentage error } \\ \text { MSE } & \text { mean squared error } \\ \text { PSF } & \text { pattern sequence-based forecasting } \\ \text { SAE } & \text { stacked auto-encoders } \\ \text { SOC } & \text { state-of-charge } \\ \text { SVM } & \text { support vector machines } \\ \text { TBATS } & \text { Trigonometric, Box-Cox, auto-regressive-moving-average, trend, and seasonality }\end{array}$




\section{References}

1. Guarnieri, M. Looking back to electric cars. In Proceedings of the 2012 Third IEEE History of Electro-Technology Conference (HISTELCON), Pavia, Italy, 5-7 September 2012; pp. 1-6.

2. Wakefield, E.H. History of the Electric Automobile Battery-Only Powered Cars; Society of Automotive Engineers: Warrendale, PA, USA, 1993.

3. Ehsani, M.; Gao, Y.; Longo, S.; Ebrahimi, K. Modern Electric, Hybrid Electric, and Fuel Cell Vehicles; CRC Press: Boca Raton, FL, USA, 2018

4. IEA. Global EV Outlook. Available online: https://www.iea.org/reports/global-ev-outlook-2019 (accessed on 8 February 2021).

5. Amini, M.H.; Kargarian, A.; Karabasoglu, O. ARIMA-based decoupled time series forecasting of electric vehicle charging demand for stochastic power system operation. Electr. Power Syst. Res. 2016, 140, 378-390. [CrossRef]

6. Zhao, W.; Dai, T.-T.; Wang, L.-C.; Lu, K.; Chen, N. Short-term Load Forecasting Considering Meteorological Factors and Electric Vehicles. IOP Conf. Ser. Mater. Sci. Eng. 2018, 439, 032114. [CrossRef]

7. Li, Y.; Huang, Y.; Zhang, M. Short-term load forecasting for electric vehicle charging station based on niche immunity lion algorithm and convolutional neural network. Energies 2018, 11, 1253. [CrossRef]

8. Zhu, J.; Yang, Z.; Guo, Y.; Zhang, J.; Yang, H. Short-term load forecasting for electric vehicle charging stations based on deep learning approaches. Appl. Sci. 2019, 9, 1723. [CrossRef]

9. Zhu, J.; Yang, Z.; Mourshed, M.; Guo, Y.; Zhou, Y.; Chang, Y.; Wei, Y.; Feng, S. Electric vehicle charging load forecasting: A comparative study of deep learning approaches. Energies 2019, 12, 2692. [CrossRef]

10. Sun, Q.; Liu, J.; Rong, X.; Zhang, M.; Song, X.; Bie, Z.; Ni, Z. Charging load forecasting of electric vehicle charging station based on support vector regression. In Proceedings of the 2016 IEEE PES Asia-Pacific Power and Energy Engineering Conference (APPEEC), Xi'an, China, 25-28 October 2016; pp. 1777-1781.

11. Lu, K.; Sun, W.; Ma, C.; Yang, S.; Zhu, Z.; Zhao, P.; Zhao, X.; Xu, N. Load forecast method of electric vehicle charging station using SVR based on GA-PSO. IOP Conf. Ser. Earth Environ. Sci. 2017, 69, 012196. [CrossRef]

12. Shi, X.; Qiu, L.; Tian, L.; Liang, M.; Yi. L. Short-term load forecasting for electric vehicle charging stations based on time series distance measuring. In Proceedings of the 2017 13th IEEE International Conference on Electronic Measurement \& Instruments (ICEMI), Yangzhou, China, 20-22 October 2017; pp. 417-423.

13. Majidpour, M.; Qiu, C.; Chu, P.; Pota, H.R.; Gadh, R. Forecasting the EV charging load based on customer profile or station measurement? Appl. Energy 2016, 163, 134-141. [CrossRef]

14. Lu, Y.; Li, Y.; Xie, D.; Wei, E.; Bao, X.; Chen, H.; Zhong, X. The application of improved random forest algorithm on the prediction of electric vehicle charging load. Energies 2018, 11, 3207. [CrossRef]

15. Cheon, S.; Kang, S.-J. An electric power consumption analysis system for the installation of electric vehicle charging stations Energies 2017, 10, 1534. [CrossRef]

16. Louie, H.M. Time-series modeling of aggregated electric vehicle charging station load. Electr. Power Compon. Syst. 2017, 45, 1498-1511. [CrossRef]

17. Buzna, L.; De Falco, P.; Khormali, S.; Proto, D.; Straka, M. Electric vehicle load forecasting: A comparison between time series and machine learning approaches. In Proceedings of the 2019 1st International Conference on Energy Transition in the Mediterranean Area (SyNERGY MED), Cagliari, Italy, 28-30 May 2019; pp. 1-5.

18. Wang, S.; Xue, G.; Ping, C.; Wang, D.; You, F.; Jiang, T. The application of forecasting algorithms on electric vehicle power load. In Proceedings of the 2018 IEEE International Conference on Mechatronics and Automation (ICMA), Changchun, China, 5-8 August 2018; pp. 1371-1375.

19. Huber, J.; Dann, D.; Weinhardt, C. Probabilistic forecasts of time and energy flexibility in battery electric vehicle charging. Appl. Energy 2020, 262, 114525. [CrossRef]

20. Gerossier, A.; Girard, R.; Kariniotakis, G. Modeling and forecasting electric vehicle consumption profiles. Energies 2019, $12,1341$. [CrossRef]

21. Arias, M.B.; Kim, M.; Bae, S. Prediction of electric vehicle charging-power demand in realistic urban traffic networks. Appl. Energy 2017, 195, 738-753. [CrossRef]

22. Lee, D.-H.; Kim, M.-S.; Roh, J.-H.; Yang, J.-P.; Park, J.-B. Forecasting of Electric Vehicles Charging Pattern Using Bayesians method with the Convolustion. IFAC-PapersOnLine 2019, 52, 413-418. [CrossRef]

23. Arias, M.B.; Bae, S. Electric vehicle charging demand forecasting model based on big data technologies. Appl. Energy 2016, 183, 327-339. [CrossRef]

24. Su, S.; Zhao, H.; Zhang, H.; Lin, X.; Yang, F.; Li, Z. Forecast of electric vehicle charging demand based on traffic flow model and optimal path planning. In Proceedings of the 2017 19th International Conference on Intelligent System Application to Power Systems (ISAP), San Antonio, TX, USA, 17-20 September 2017; pp. 1-6.

25. Moon, H.; Park, S.Y.; Jeong, C.; Lee, J. Forecasting electricity demand of electric vehicles by analyzing consumers' charging patterns. Transp. Res. Part D Transp. Environ. 2018, 62, 64-79. [CrossRef]

26. Wang, H.; Zhang, Y.; Mao, H. Load Forecasting Method of EVs Based on Time Charging Probability. In Proceedings of the 2018 International Conference on Power System Technology (POWERCON), Denver, CO, USA, 16-19 April 2018; pp. 1731-1735. 
27. Mao, M.; Yue, Y.; Chang, L. Multi-time scale forecast for schedulable capacity of electric vehicle fleets using big data analysis. In Proceedings of the 2016 IEEE 7th International Symposium on Power Electronics for Distributed Generation Systems (PEDG), Vancouver, BC, Canada, 27-30 June 2016; pp. 1-7.

28. Yan, J.; Zhang, J.; Liu, Y.; Lv, G.; Han, S.; Alfonzo, I.E.G. EV charging load simulation and forecasting considering traffic jam and weather to support the integration of renewables and EVs. Renew. Energy 2020, 159, 623-641. [CrossRef]

29. Tat, T.H.C.; Fränti, P. Real-time Electric Vehicle Load Forecast to Meet Timely Energy Dispatch. In Proceedings of the 2018 IEEE International Conference on Service Operations and Logistics, and Informatics (SOLI), Zhengzhou, China, 11-13 October 2018; pp. 148-153.

30. Gomez-Quiles, C.; Asencio-Cortes, G.; Gastalver-Rubio, A.; Martinez-Alvarez, F.; Troncoso, A.; Manresa, J.; Riquelme, J.C.; Riquelme-Santos, J.M. A novel ensemble method for electric vehicle power consumption forecasting: Application to the spanish system. IEEE Access 2019, 7, 120840-120856. [CrossRef]

31. Choi, S.; Sohn, H.G.; Kim, S. A study on electricity demand forecasting for electric vehicles in KOREA. Korean Data Inf. Sci. Soc. 2018, 29, 1137-1153.

32. Tan, B.; Chen, H. Multi-objective energy management of multiple microgrids under random electric vehicle charging. Energy 2020, 208, 118360. [CrossRef]

33. Al-Ogaili, A.S.; Hashim, T.J.T.; Rahmat, N.A.; Ramasamy, A.K.; Marsadek, M.B.; Faisal, M.; Hannan, M.A. Review on scheduling, clustering, and forecasting strategies for controlling electric vehicle charging: Challenges and recommendations. IEEE Access 2019, 7, 128353-128371. [CrossRef]

34. Kim, Y.; Son, H.G.; Kim, S. Short term electricity load forecasting for institutional buildings. Energy Rep. 2019, 5, 1270-1280. [CrossRef]

35. De Livera, A.M.; Hyndman, R.J.; Snyder, R.D. Forecasting time series with complex seasonal patterns using exponential smoothing. J. Am. Stat. Assoc. 2011, 106, 1513-1527. [CrossRef]

36. Box, G.; Jenkins, G.; Reinsel, G.; Ljung, G. Time Series Analysis, Control, and Forecasting; John Wiley \& Sons: Hoboken, NJ, USA, 2015.

37. Demuth, H.B.; Beale, M.H.; De Jess, O.; Hagan, M.T. Neural Network Design; Martin Hagan: Stillwater, OK, USA, 2014.

38. Hochreiter, S.; Schmidhuber, J. Long short-term memory. Neural Comput. 1997, 9, 1735-1780. [CrossRef] [PubMed]

39. Ministry of Environment, Republic of Korea. Available online: http:/ / eng.me.go.kr (accessed on 8 February 2021). 